Punjab University Journal of Mathematics (2021),53(5),307-318

https://doi.org/1.52280/pujm.2021.5300501

\title{
Finite Difference Analysis of Unsteady Heat and Flow of an Incompressible Third Grade Fluid
}

\author{
I Nayak \\ Department of Mathematics, \\ VSSUT, Burla, Odisha, India, \\ Email: itii.nayak@gmail.com
}

Received: 21 February, 2020 / Accepted: 25 March, 2021 / Published online: May 26, 2021

\begin{abstract}
An unsteady flow and heat transfer problem with viscous dissipation of a third order fluid placed within two long parallel flat porous walls is studied in present work. The governing equations are non- dimensionalized and finally a non-linear coupled system of partial differential equation is obtained. An approximated solution is obtained using finite difference method of fully implicit form. With help of high speed MATLAB programming numerical solution is procured and presented graphically. Investigation is made on effect of different physical parameters on flow and heat profile. The notable finding in the current work is that for smaller values of visco-elastic parameter $\alpha$, the velocity rises with raising the values of $\alpha$. But for larger visco-elastic parametric values of $\alpha$, a reversed effect is seen on velocity field. Also with the increase of viscous-dissipation parameter, more viscous-dissipation heat generated that increases the temperature field.
\end{abstract}

MSC2020- Subject Classification Codes: 65M06; 65N22; 76A05

Key Words: Third grade fluid, channel flow, Damped-Newton method, porous plates.

\section{INTRODUCTION}

Because of wide application in industry and engineering, the research on non-Newtonian fluids has been emphasized. To analyze the complex properties of non-Newtonian fluids many models and constitutive equations have been put forward. Among those fluid of second grade is the simplest model which is capable to forecast the normal stress differences. Several studies has been done on this model like Teipel(1988), Sharma and Nageswar Row(1998), Mohapatra et al.(2001), Ariel(2002), Hayat et al.(2004), Vajravelu and Rollins(2004), Tan(2005), Khan et al(2017).

However, it is unable to predict phenomenon of shear thinning and thickening, which is observed in most non-Newtonian fluids described by Joseph and Fosdick(1973), Beavers and Joseph(1975). This model of third grade is one special class of fluid that include such characteristic of visco-elastic fluids. Which inspire many researchers in this direction 
as Fosdick and Rajagopal(1980), Kaloni and siddique(1987), Erdogan(1995),Ariel(2002), Hayat et al.(2002), Okaya(2008), Hayat et al.(2008), Nayak et al.(2012), Sahoo and Poncet(2011), Mukhopadhya(2013). Recently, Hayat et al.(2015) have studied MHD axisymmetric third grade fluid flow in a stretching cylinder. They use suitable transformation and turn the partial differential equation into ordinary differential equation and then the system of equation is solved by homotopy analysis method. Ahmed et al.(2017) have analysed heat transfer of a third grade fluid flow within parallel plates and they get results using variation of parameter method. Akinshilo(2017) inspected steady flow and heat transfer of a fluid of grade three in a porous medium and solved arising non-linear differential equation using adomain decomposition method.

In present paper the time depended channel flow and energy equation of the fluid of grade three progress within two infinitely long porous plates is discussed. Where the bottom plate instantly starts in motion with velocity $\mathrm{F}(\mathrm{t})$, that vary with time. Then implicit FDM is used to acquire a system of non-linear algebraic equations and then numerical solution is obtained by damped Newton method. Moreover the employed scheme is sustainable for both large and small elastic parameter values. In addition recast of entire problem is not necessary for any change in boundary condition unlike the earlier analytical methods. So the present investigation is meaningful because of its increasing application in technology and interesting methodical mathematical features.

The succession of work is arranged as: Sec. 2 construction of problem, Sec. 3 discussion of solution procedure with convergence of employing scheme, Sec. 4 explanation of the effect of different physical parameters on velocity and temperature profile through graphs, Sec. 5 culminates the study.

\section{Formulation OF PROBLEM}

Here lower plate is considered as $\mathrm{x}$-axis and $\mathrm{y}$-axis is taken normal to it. The plates are considered to be infinite long in either direction of $x^{\prime}$-axis. The upper plate is of length $L$ and $V>0$ indicates the suction velocity near the plate. The components of velocity $u^{\prime}$ and $v^{\prime}$ along the axis $x^{\prime}$ and $y^{\prime}$ respectively consistent with the equation of continuity

$$
u^{\prime}=u^{\prime}\left(y^{\prime}, t^{\prime}\right), v^{\prime}=V
$$

The stress $P$ in the third grade fluid Fosdick and Rajagopal (1980) is

$P=-p I+\mu A_{1}+\alpha_{1} A_{2}+\alpha_{2} A_{1}^{2}+\beta_{1} A_{3}+\beta_{2}\left(A_{1} A_{2}+A_{2} A_{1}\right)+\beta_{3}\left(\operatorname{tr} A_{1}^{2}\right) A_{2}$

Where

$$
\begin{aligned}
& \mu=\text { viscosity } \\
& \alpha_{1}, \alpha_{2}=\text { second grade elastic parameter } \\
& \beta_{1}, \beta_{2}, \beta_{3}=\text { third grade elastic parameter } \\
& p=\text { pressure } \\
& I=\text { identity tensor } \\
& A_{n}=\text { kinematic tensor, defined as } \\
& A_{0}=I, A_{1}=\nabla u+(\nabla u)^{T} \text { and } \\
& A_{n}=\frac{\partial}{\partial t} A_{n-1}+\nabla u \cdot A_{n-1}+(\nabla u)^{T} . A_{n-1} \text { for } n \geq 2 \\
& \text { where } \frac{\partial}{\partial t} \text { is the material timederivative. By Fosdick and Rajagopala (1980), for a ther- } \\
& \text { modynamically compatible fluid flows satisfy the Clausius-Duhem inequality, locally at }
\end{aligned}
$$


rest with specific Helmholtz free energy is minimum requires that $\mu \geq 0 ; \beta_{1}=\beta_{2}=0 ; \alpha_{1} \geq 0 ;\left|\alpha_{1}+\alpha_{2}\right| \leq \sqrt{24 \mu \beta_{3}}$

Therefore, for a thermodynamically compatible fluid of grade three the velocity equation takes the form

$$
\rho\left(\frac{\partial u^{\prime}}{\partial t^{\prime}}+V \frac{\partial u^{\prime}}{\partial y^{\prime}}\right)=\mu \frac{\partial^{2} u^{\prime}}{\partial y^{\prime 2}}+\alpha_{1} \frac{\partial^{3} u^{\prime}}{\partial y^{\prime 2} \partial t^{\prime}}+6 \beta_{3}\left(\frac{\partial u^{\prime}}{\partial y^{\prime}}\right)^{2} \frac{\partial^{2} u^{\prime}}{\partial y^{\prime 2}}+\alpha_{1} V \frac{\partial^{3} u^{\prime}}{\partial y^{\prime 3}}
$$

For the following subjected initial and boundary conditions

$$
\begin{array}{r}
t^{\prime}=0: u^{\prime}=0, \forall y^{\prime} \\
t^{\prime}>0: u^{\prime}=F(t) \text { for } y=0 \\
u^{\prime}=0 \text { for } y^{\prime}=L
\end{array}
$$

Where $F(t)=A \sin \left(w^{\prime} t^{\prime}\right)$

The energy equation for the thermodynamically compatible third grade fluid with viscous dissipation is

$$
\begin{aligned}
\rho c_{p}\left(\frac{\partial \theta^{\prime}}{\partial t^{\prime}}+V \frac{\partial \theta^{\prime}}{\partial y^{\prime}}\right) & =k \frac{\partial^{2} \theta^{\prime}}{\partial y^{\prime 2}}+\mu\left(\frac{\partial u^{\prime}}{\partial y^{\prime}}\right)^{2}+\alpha_{1}\left(\frac{\partial^{2} u^{\prime}}{\partial y^{\prime} \partial t^{\prime}}\right)\left(\frac{\partial u^{\prime}}{\partial y^{\prime}}\right) \\
& +\alpha_{1} V\left(\frac{\partial^{2} u^{\prime}}{\partial y^{\prime 2}}\right)\left(\frac{\partial u^{\prime}}{\partial y^{\prime}}\right)+2 \beta_{3}\left(\frac{\partial u^{\prime}}{\partial y^{\prime}}\right)^{4}
\end{aligned}
$$

For the following subjected initial and boundary conditions

$$
\begin{array}{r}
t^{\prime}=0: \theta^{\prime}=0 \forall y^{\prime} \\
t^{\prime}>0: \theta^{\prime}=\theta_{2} \text { for } y^{\prime}=0 \\
\theta^{\prime}=0 \text { for } y^{\prime}=L
\end{array}
$$

where $=$ density of fluid, $\mu=$ viscosity, $c_{p}=$ specific heat, $k=$ thermal conductivity and $\theta^{\prime}=$ temperature.

Considering the non-dimensional parameters as follows:

$$
\begin{array}{r}
y=\frac{y^{\prime}}{\sqrt{\vartheta T}}, t=\frac{t^{\prime}}{T}, u=\frac{u^{\prime}}{A}, \theta=\frac{\theta^{\prime}-\theta_{1}}{\theta_{2}-\theta_{1}}, \omega=\omega^{\prime} T, R=\frac{V \sqrt{T}}{\sqrt{\vartheta}}, \alpha=\frac{\alpha_{1}}{\vartheta T \rho}, \gamma=\frac{6 \beta_{3} A^{2}}{\rho \vartheta^{2} T} \\
p_{r}=\frac{\vartheta \rho c_{p}}{k}, E_{c}=\frac{A^{2}}{c_{p}}\left(\theta_{2}-\theta_{1}\right)
\end{array}
$$

where R - Reynolds number, $\alpha$ - visco-elastic parameter, $\gamma$ - third grade elastic parameter, $\mathrm{Pr}$ - Prandtl number, Ec - viscous dissipation parameter, $\vartheta$-kinematic coefficient of viscosity, $\mathrm{A}=$ constant.

Then the momentum equation in non-dimensionalised form can be written as

$$
\left(\frac{\partial u}{\partial t}+R \frac{\partial u}{\partial y}\right)=\frac{\partial^{2} u}{\partial y^{2}}+\alpha \frac{\partial^{3} u}{\partial y^{2} \partial t}+\gamma\left(\frac{\partial u}{\partial y}\right)^{2} \frac{\partial^{2} u}{\partial y^{2}}+R \alpha \frac{\partial^{3} u}{\partial y^{3}}
$$


with the subjected conditions as

$$
\begin{array}{r}
t=0: u=0, \forall y \\
t>0: u=\sin \omega t, \text { for } y=0 \\
u=0, \text { for } y=1
\end{array}
$$

The energy equation is obtained in non-dimensionalized form can be written as

$$
\begin{aligned}
\left(\frac{\partial \theta}{\partial t}+R \frac{\partial \theta}{\partial y}\right) & =\frac{1}{p_{r}} \frac{\partial^{2} \theta}{\partial y^{2}}+E_{c}\left(\frac{\partial u}{\partial y}\right)^{2}+\alpha E_{c}\left(\frac{\partial^{2} u}{\partial y \partial t}\right)\left(\frac{\partial u}{\partial y}\right) \\
& +\alpha R E_{c}\left(\frac{\partial^{2} u}{\partial y^{2}}\right)\left(\frac{\partial u}{\partial u}\right)+\frac{\gamma E_{c}}{3}\left(\frac{\partial u}{\partial y}\right)^{4}
\end{aligned}
$$

With the subjected conditions as

$$
\begin{array}{r}
t=0: \theta=0, \text { for all } y \\
t>0: \theta=1, \text { for } y=0 \\
\theta=0, \text { for } y=1
\end{array}
$$

The above non-linear coupled system of partial differential equation is worked out along the relevant initial and boundary conditions using FDM. Then the numerical solution of the system is obtained using damped-Newton method explained in C., De Boor (1980).

\section{Solution Method}

The velocity equation (2.7) with the equation (2.9) is descretized by finite difference method with an invariable mesh step $h$ and time step $\Delta t$, it so generated as

$$
\left(y_{j}, t_{j}\right)=(i h, j \Delta t), i=0,1,2, \ldots, N+1, j=0,1,2, \ldots, M
$$

The time step is taken as $t_{M}=M \Delta t$ for some suitable $M$ where the velocity and temperature field is to be computed. To implement the boundary conditions at infinity, $N$ is so chosen that the modulus of difference between consecutive results obtained at $((N+1) h, j \Delta t)$ and $((N+2) h, j \Delta t)$ is less than an assigned error tolerance $\epsilon$. A manageable algorithm stated in Jain(1984) is followed here to set $N$ approximately. Following notations and finite difference approximations of the derivatives are taken at distinct nodes as $d_{i j} \mathrm{~s}$.

Notations:

$$
\begin{aligned}
& d_{1 i}^{j}=u_{i+1}^{j}-u_{i-1}^{j} \\
& d_{2 i}^{j}=u_{i+1}^{j}-2 u_{i}^{j}+u_{i-1}^{j} \\
& d_{3 i}^{j}=-u_{i-2}^{j}+2 u_{i-1}^{j}-2 u_{i+1}^{j}+u_{i-1}^{j} \\
& d_{3 i}^{\prime j}=-3 u_{i-1}^{j}+10 u_{i}^{j}-12 u_{i+1}^{j}+6 u_{i+2}^{j}-u_{i+3}^{j} \\
& d_{3 i}^{\prime \prime}=u_{i-3}^{j}-6 u_{i-2}^{j}+12 u_{i-1}^{j}-10 u_{i}^{j}+3 u_{i+1}^{j}
\end{aligned}
$$


The finite difference approximation at nodes $(i h, j \Delta t)$ are written as

$$
\begin{aligned}
& \frac{\partial u}{\partial t} \approx \frac{u_{i}^{j+1}-u_{i}^{j}}{\Delta t} \\
& \frac{\partial u}{\partial y} \approx \frac{d_{1 i}^{j+1}+d_{1 i}^{j}}{4 h} \\
& \frac{\partial^{2} u}{\partial y^{2}} \approx \frac{d_{2 i}^{j+1}+d_{2 i}^{j}}{2 h^{2}} \\
& \frac{\partial^{3} u}{\partial y^{3}} \approx \frac{d_{3 i}^{j+1}+d_{3 i}^{j}}{2 h^{3}} \\
& \frac{\partial^{3} u}{\partial y^{2} \partial t} \approx \frac{d_{2 i}^{j+1}-d_{2 i}^{j}}{h^{2} \Delta t} \text { for } i=1,2,3, \ldots, N j=0,1,2, \ldots, M
\end{aligned}
$$

$\operatorname{At}(1, j \Delta t)$ and $(N, j \Delta t), \frac{\partial^{3} u}{\partial y^{3}}$ is descretized respectively by $\frac{d_{3 i}^{\prime j+1}+d_{3 i}^{\prime}}{2 h^{3}}$ and $\frac{d_{3 i}^{\prime \prime j+1}+d_{3 i}^{\prime \prime} j}{2 h^{3}}$. Using the above expressions the equations of velocity and energy with prescribed conditions are written in discretized form. With implementation of the above said notations in (3.1), the discretized flow equation becomes

$$
\begin{aligned}
\frac{u_{i}^{j+1}-u_{i}^{j}}{\Delta t}+R \frac{d_{1 i}^{j+1}+d_{1 i}^{j}}{4 h} & =\frac{d_{2 i}^{j+1}+d_{2 i}^{j}}{2 h^{2}}+\alpha \frac{d_{2 i}^{j+1}-d_{2 i}^{j}}{h^{2} \Delta t} \\
& +R \alpha \frac{d_{3 i}^{j+1}+d_{3 i}^{j}}{2 h^{3}}+\frac{\gamma}{32 h^{4}}\left(d_{1 i}^{j+1}+d_{1 i}^{j}\right)^{2}\left(d_{2 i}^{j+1}+d_{2 i}^{j}\right)
\end{aligned}
$$

For $i=1,2,3, \ldots ., N-1, j=0,1, \ldots, M$.

With the discretized subsequent conditions

$$
\begin{aligned}
& u_{i}^{0}=0, i=0,1, \ldots, N+1 \\
& u_{0}^{j}=\sin \omega j \Delta t \\
& u_{N+1}^{j}=0, j=1, \ldots, M
\end{aligned}
$$

The energy equation is discretized as

$$
\begin{aligned}
& \frac{\theta_{i}^{j+1}-\theta_{i}^{j}}{\Delta t}+\frac{R}{4 h}\left[\left(\theta_{i+1}^{j+1}-\theta_{i-1}^{j+1}\right)+\left(\theta_{i+1}^{j}-\theta_{i-1}^{j}\right)\right]= \\
& \frac{1}{p_{r} 2 h^{2}}\left[\left(\theta_{i+1}^{j+1}-2 \theta_{i}^{j+1}+\theta_{i-1}^{j+1}\right)+\left(\theta_{i+1}^{j}-2 \theta_{i}^{j}+\theta_{i-1}^{j}\right)\right]+E_{c}\left(\frac{d_{1 i}^{j+1}+d_{1 i}^{j}}{4 h}\right)^{2} \\
& +\frac{\gamma E_{c}}{3 \times 16^{2} \times h^{4}}\left(d_{1 i}^{j+1}+d_{1 i}^{j}\right)^{4}+\frac{E_{c} \alpha}{8 h^{2} \Delta t}\left(d_{1 i}^{j+1}+d_{1 i}^{j}\right)\left(d_{1 i}^{j+1}-d_{1 i}^{j}\right) \\
& +\frac{\alpha R E_{c}}{8 h^{3}}\left(d_{1 i}^{j+1}+d_{1 i}^{j}\right)\left(d_{2 i}^{j+1}+d_{2 i}^{j}\right)
\end{aligned}
$$


With the discretized subsequent conditions

$$
\begin{aligned}
& \theta_{i}^{0}=0, i=0,1,2, \ldots, N+1 \\
& \theta_{0}^{j}=1, \text { and } \\
& \theta_{N+1}^{j}=0, j=1,2, \ldots, M
\end{aligned}
$$

On rearrangement of energy equation, it is converted into a tri-diagonal system of equations. Which is then worked out applying appropriate form of Gaussian elimination method with the help of initial velocities which are obtained again by solving the tri-diagonal system for velocity formed by making the parameter $\alpha$ and $\gamma$ become zero in constitutive equation of velocity given in eq.(3.2). Similarly initial choice of temperature is made converting the energy equation into a tri-diagonal system by making the parameter $E_{c}$ and alpha become zero and then solved it using the exponentially fitted scheme by Morton(1996).

Then the non-linear system equation for velocity (3.2) along with (3.3) is resolved by using above mentioned numerical method. For this the residuals $\left(R_{i}, i=0,1, \ldots, N\right)$ and the non-zero components of Jacobian matrix $\left(\frac{\partial R_{i}}{\partial u_{j}}\right), i=1,2,3, \ldots, N$ and $j=$ $1,2,3, \ldots, M$ for the system of equations are calculated.

Then velocity field obtained after every iteration is used in the temperature equation and the thermal transitions is studied. In aforementioned iteration scheme a new approximation for velocity being accepted as $f\left(x^{m}+\frac{h}{2^{i}}\right)$ for some suitable i for which it must satisfy $\left\|f\left(x^{m}+\frac{h}{2^{i}}\right)\right\|_{2}<\left\|f\left(x^{m}\right)\right\|_{2}$. Otherwise the failure occur. That ensure that there must be decrease in residual error after every iteration and hence the above used scheme is convergent and efficient. The above procedure is done using MATLAB coding and solutions obtained are discussed graphically in concluding section.

\section{RESUlts AND Discussion}

The study reveals the flow and heat analysis of a third order fluid passed between two horizontally placed two porous walls. Results are plotted graphically in fig- 1 to fig-16 for different choices of physical parameters.

Fig-1, 2 depicts that velocity profile decline for an increase in $\alpha$ that is enhancing the visco-elastic parameter $\alpha$ increases visco-elasticity of the fluid that is turn down velocity of the fluid. Where as a noticeable opposite effect is seen for temperature profile.

Fig-3, 4 it is noticed here that for small elastic parameter values of $\alpha$, an opposite effect on velocity. This depicts that a small increase in visco-elastic parameter increases momentum boundary level thickness. It is also noted that near boundary, the effect of elastic parameter is some less than that farther away from the boundary. While an opposite effect is seen for thermal boundary layer.

Fig-5,6 depicts the consequence of third grade elastic parameter $\gamma$ for the flow and temperature field. It is noted here the flow profile decreases gradually with the rise of the non-Newtonian parameter $\gamma$ unlike the second order parameter $\alpha$ where velocity falls suddenly. Whereas temperature field rises on increase of elastic parameter $\gamma$ and the effect is seen pronounced to farther away from the thermal boundary. 

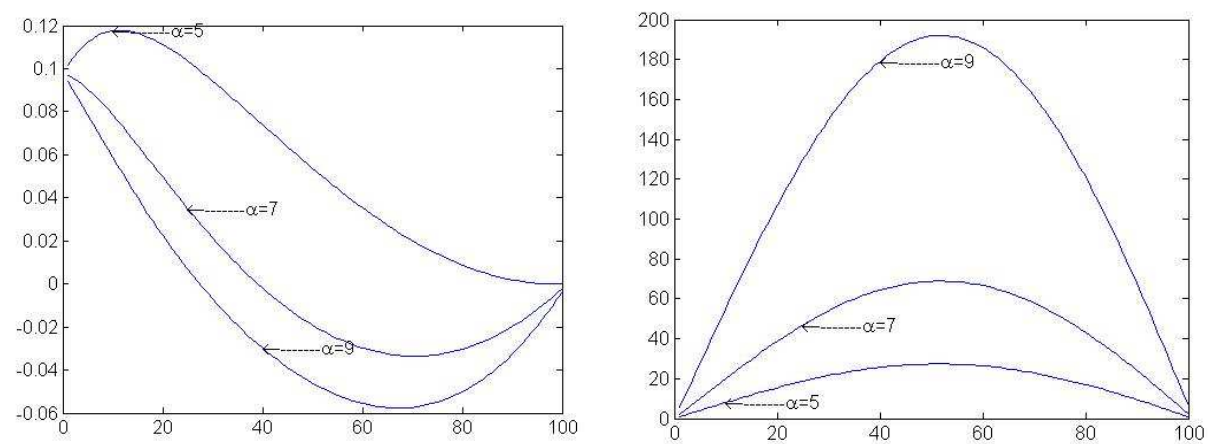

Figure 1: Effect of $\alpha$ on Velocity field ( $\gamma=$ Figure 2: Effect of $\alpha$ on Temp. field $(\gamma=$ $3, R e=7, w=1, n=100, a n=1, h l=3, R e=7, w=1, n=100$, an $=1, h l=$ $0.001, d t=0.01, p r=0.3, E c=.7) \quad 0.001, d t=0.01, p r=0.3, E c=.7)$
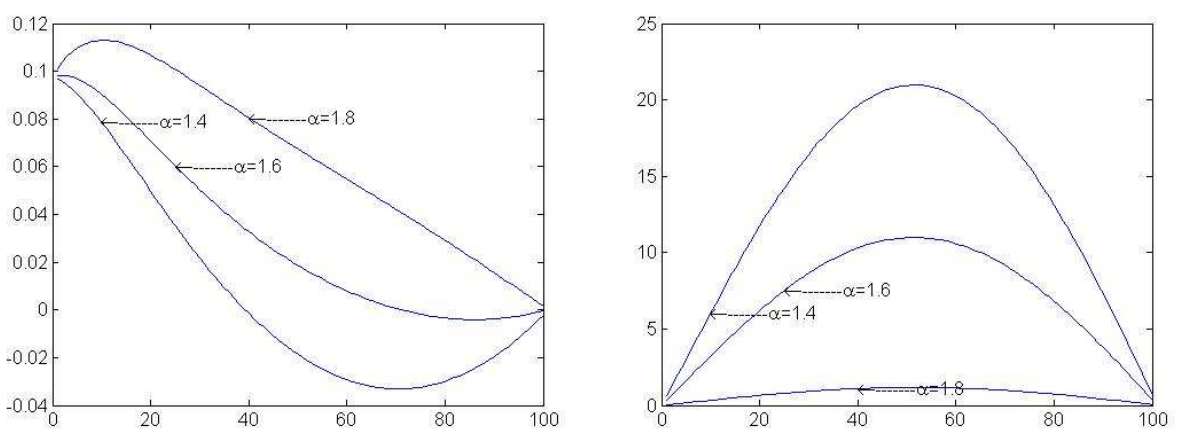

Figure 3: Effect of $\alpha$ on Velocity field ( $\gamma=$ Figure 4: Effect of $\alpha$ on Temp. field $(\gamma=$ $3, R e=8, w=1, n=100, a n=1, h l=3, R e=8, w=1, n=100$, an $=1, h l=$ $.001, d t=.01, p r=.3, E c=.7)$ $0.001, d t=0.01, p r=0.3, E c=0.7)$

From fig-7, 8 It is noticed for small of elastic parametric values of $\gamma$ the impact on flow and heat field is quite similar as in case of larger value of above elastic parameter. But the result is not so pronounced for both the fields.

Fig-9, 10 depicts the reflex of Reynolds number $\left(R_{e}>1\right)$ on flow and temperature field. Which shows when $R_{e}$ increases, viscous force of the fluid gradually increases that causes decrease in both velocity and temperature profile at all points of the fluid. But velocity functions are concave in nature and temperature functions are convex in nature. It is also noted that the consequence of $R_{e}$ on temperature field diminished farther away to plate. 

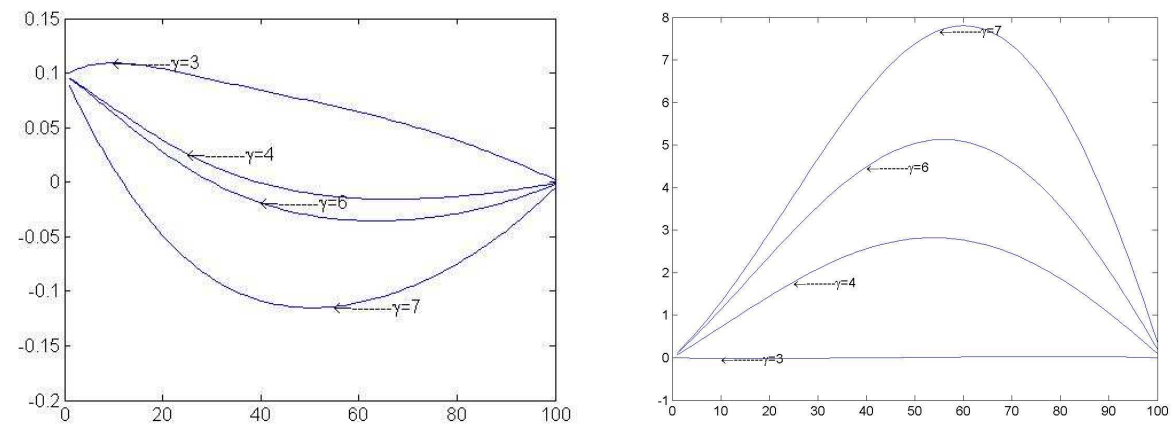

Figure 5: Effect of $\gamma$ on Velocity field ( $\alpha=$ Figure 6: Effect of $\gamma$ on Temp. field ( $\alpha=$ $3, R e=8, w=1, n=100$, an $=1, h l=3, R e=8, w=1, n=100$, an $=1, h l=$ $.001, d t=.01, p r=.3, E c=.7)$ $0.001, d t=0.01, p r=0.3, E c=0.7)$
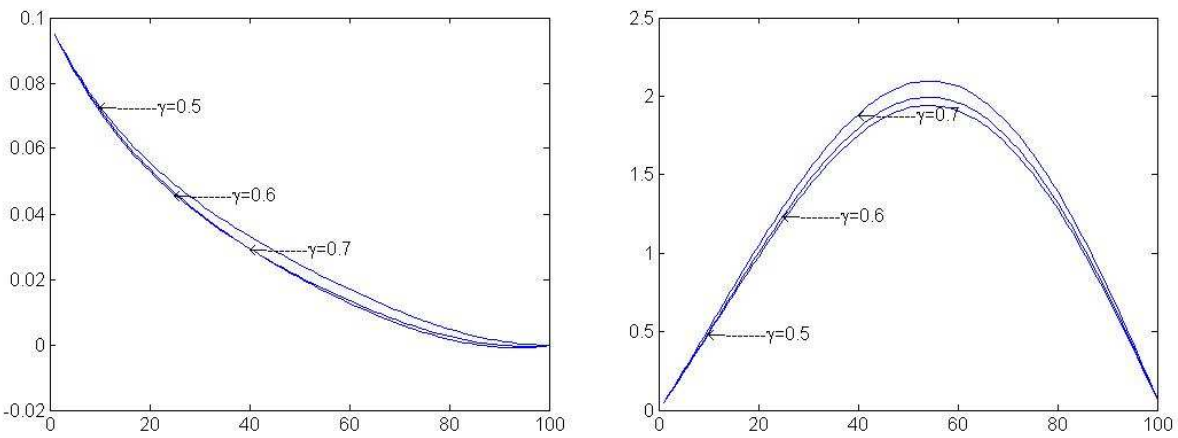

Figure 7: Effect of $\gamma$ on Velocity field ( $\alpha=$ Figure 8: Effect of $\gamma$ on Temp. field ( $\alpha=$ $2, R e=8, w=1, n=100$, an $=1, h l=2, R e=8, w=1, n=100$, an $=1, h l=$ $.001, d t=.01, p r=.3, E c=.7)$ $0.001, d t=0.01, p r=0.3, E c=0.7)$

From fig-11, 12 it is perceived here that both heat and fluid flow become slower with increase in $\left(R_{e}<1\right)$ and the results on velocity field is seems to be linear. That influence on flow and heat field continuously diminished far away from boundary.

Fig-13 shows the result of prandtl number $\left(P_{r}\right)$ on temperature field. It is noticed here as the values of $\left(P_{r}\right)$ increases, the heat flow profile decreases nearby surface of the plate and gradually rises away from the plate and results a cross over temperature profile.

Fig-14 depicts when $P_{r}<1$ temperature flow profile becomes smaller for increase of prandtl number $\left(P_{r}\right)$. While a pronounced effect is seen near the plate and the effect gradually diminished towards away from the plate. 

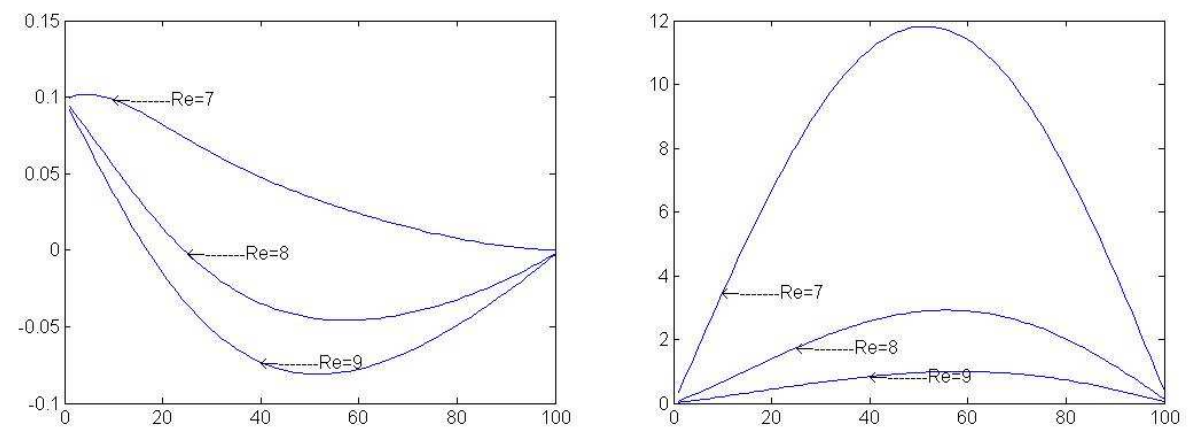

Figure 9: Effect of $R_{e}$ on Velocity field ( $\alpha=$ Figure 10: Effect of $R_{e}$ on Temp. field ( $\alpha=$ $3, \gamma=3, w=1, n=100$, an $=1, h l=3, \gamma=3, w=1, n=100$, an $=1, h l=$ $0.001, d t=0.01, p r=0.3, E c=0.5) \quad 0.001, d t=0.01, p r=0.3, E c=0.5)$
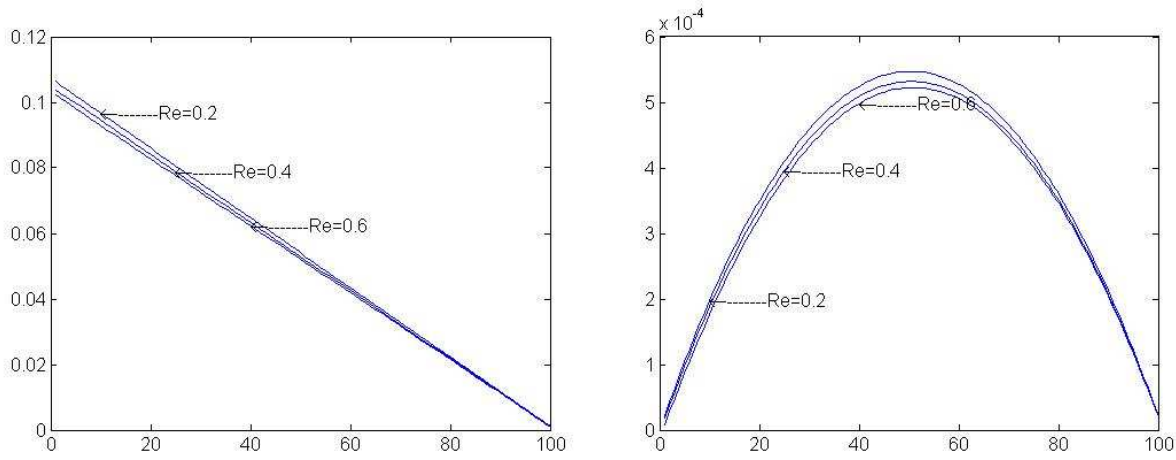

Figure 11: Effect of $R_{e}$ on Velocity field ( $\alpha=$ Figure 12: Effect of $R_{e}$ on Temp. field ( $\alpha=$ $0.01, \gamma=1, w=1, n=100$, an $=1, h l=0.01, \gamma=1, w=1, n=100$, an $=1, h l=$ $0.001, d t=0.01, p r=0.3, E c=0.5) \quad 0.001, d t=0.01, p r=0.3, E c=0.5)$

Fig-15 recites the result of viscous dissipation parameter $\left(E_{c}\right)$ on heat flow profile. It is considered as association between kinetic energy of the flow and boundary layer difference enthalpy. This shows the change of kinetic energy by work done opposed to the viscous fluid stress. So when $\left(E_{c}\right)$ increases, more the viscous dissipation heat generated cause a rise in the temperature field.

Finally fig-16 recite the effect of Eckert number $\left(E_{c}\right)<1$ on heat flow field. That impact is quite similar to previous result. 

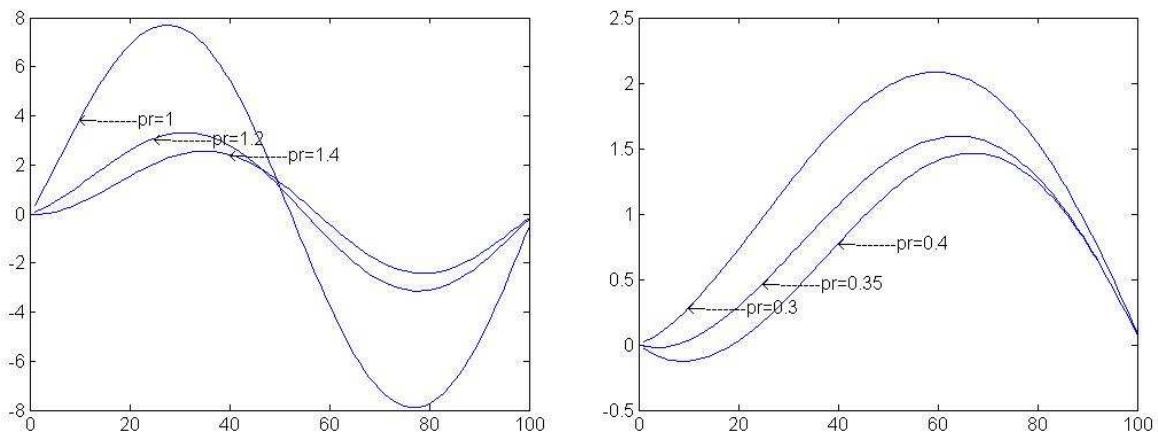

Figure 13: Effect of $P_{r}$ on Temp. $\left(\alpha=3, \gamma=\right.$ Figure 14: Effect of $P_{r}$ on Temp. $(\alpha=3, \gamma=$ $3, R e=9, n=100, w=1, E c=0.5, d t=3, R e=9, n=100, w=1, E c=0.5, d t=$ $0.01)$ $0.01)$
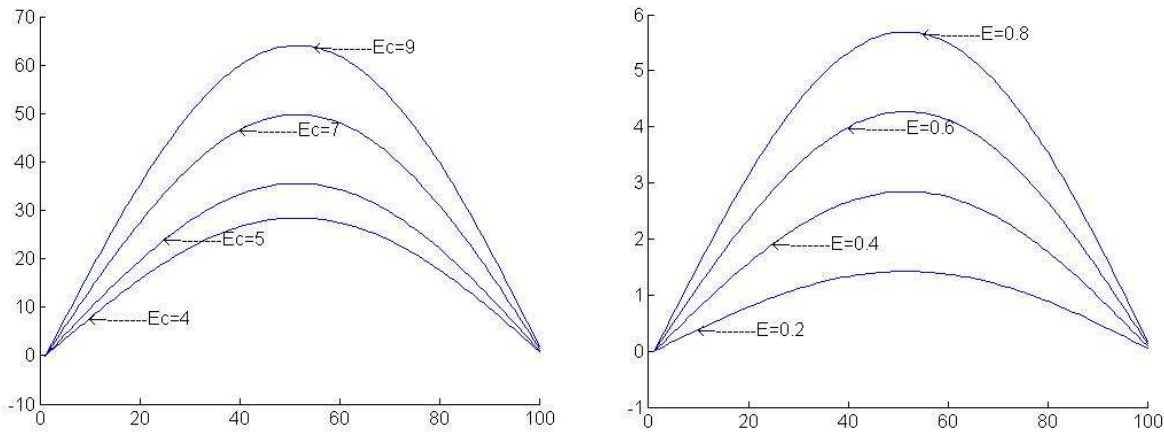

Figure 15: Effect of $E_{c}$ on Temp. $\left(\alpha=2, \gamma=\right.$ Figure 16: Effect of $E_{c}$ on Temp. $(\alpha=$ $2, R e=7, w=1, P_{r}=0.5$, an $=1, h l=0.01, \gamma=2, R e=7, w=1, P_{r}=0.5$, an $=$ $0.001, d t=0.01)$ $1, h l=0.001, d t=0.01$

\section{CONCLUSION}

A numerical study is done on an unsteady heat and fluid flow problem of an incompressible third grade fluid within a porous channel with viscous dissipation. A fully implicit FDM is employed to obtain a system of algebraic equations and finally numerical solutions are obtained using damped-Newton method. The method employed here has advantage that it is sustainable for all elastic parameter values and need not require repeated evaluation of entire problem for every change in boundary conditions. The results obtained are illustrated graphically and consequence of parameters on velocity and heat profile are discussed. The 
significant results obtained in present investigation is that velocity decreases for both viscoelastic parameters with increase of large elastic parametric values. While thermal boundary layer increases substantially for both the cases. But for smaller values of elastic parameter it is found that velocity rises with rise in parameter value $\alpha$ and decline for elastic parameter $\gamma$. Moreover, temperature profile increases due to more viscous dissipation energy generated when Eckert number $E c$ increases gradually.

\section{REFERENCES}

[1] P.D. Ariel, On exact solution to flow problems of a second grade fluid through two parallel porous boundaries, Int. J. Engineering Science 40, No. 8 (2001) 913-941.

[2] P.D. Ariel, Flow of third grade fluid through a porous flatchannel, Int. J. Engineering Science 41, No. 8 (2002) 1267-1285.

[3] N. Ahemed, S. U. Jan, U. Khan and S.T. Mohyudin, Heat transfer analysis of a third grade fluid flow between parallel plates: Analytical solutions, Int J of Applied and Computational Mathematics 3, No. 2(2017) 579589.

[4] A.T. Akinshilo, Steady flow and heat transfer analysis of third grade fluid with porous medium and heat generation, Engineering Science and Technology, an International Journal 20, No.6 (2017) 1602-1609.

[5] G.S. Beavers, and D.D. Joseph, The rotating rod viscometer, J. Fluid mech. 69, No.3 (1975), 475-511.

[6] S.D. Conte, and C. De Boor, Elementary numerical analysis an algorithmic approach, McGraw-Hill, Inc, 1980.

[7] M.E. Erdogan, Plane surface suddenly set in motion in a non-Newtonian fluid, Acta mech. 108, No.1-4 (1995), 179-187.

[8] R.L. Fosdick, and, K.R. Rajagopal, Thermodynamics and stability of fluids of third grade, Proc. of Royal society London-A 369, (1980) 351-377.

[9] T.Hayat, S. Nadeem, and A.M. Siddiqui, Fluctuating flow of a third order fluid on a porous plate in a rotating medium, Int.J. of non-linear Mech. 36, No.6 (2002) 901-916.

[10] T. Hayat, M.I. Hameed, S. Asghar, and A.M. Siddiqui, Some steady MHD flows of a second grade fluid, Meccanica 39, No.4 (2004).345-355.

[11] T. Hayat, N. Ahmed, and M. Sajid, Analytic solution for MHD flow of a third grade fluid in a porous channel, J. of comp. and app. Maths 214 , No.2 (2008) 572-582.

[12] T. Hayat, A. Shafiq, and A.Alsaedi, MHD axisymmetric flow of a third grade fluid by stretching cylinder, 54, No.2 (2015) 205-212.

[13] M.K. Jain, Numerical solution of differential equations, 2ED, Wiliy Eastern 1984.

[14] D.D. Joseph, and R.L. Fosdick, The free surface on a liquid between cylinders rotating at different speeds, Arch. Rat. Mech. Anal. 49, No.5 (1973) 321-401.

[15] P. N. Kaloni, and A.M. Siddique, A note on the flow of a visco-elastic fluid between eccentric disc, J. non-Newtonian fluid Mech. 26, No.1 (1987) 125-133.

[16] M. Khan, M. ur Rahman and M. Mazur, Axisymmetric flow and heat transfer to modified second grade fluid over a radially strtching sheet, Results in physics 7, No.1 (2017) 878-889.

[17] P. Mohapaatra, S. Padhy, and S. Tripathy, Finite difference analysis of a second order liquid past an infinite vertical plate in the presence of a magnetic field, Int. J. of mech. and Engg. 6, No.1 (2001) 71-90.

[18] S. Mukhopadyaya, Slip effects on MHD boundary layer flow over an exponentially stretching sheet with suction/blowing and thermal radiation, Ain Shams Engineering Journal 4, No.3 (2013) 485-491.

[19] K.W. Morten, Numerical solution of convection diffusion problems, Springer, 1995.

[20] I. Nayak, A.K. Nayak, and S. Padhy, Numerical solution for the flow and heat transfer of a third grade fluid past a porous vertical plate, Adv. St. in the The. Phy. 6, No.13 (2012) 615-624.

[21] I. Nayak, S. Padhy, Unsteady MHD flow analysis of a third grade fluid between two porous plates, J. of the Orissa math. Society 31, No.1 (2012) 83-96.

[22] S. S. Okaya, On the transition for a generalized couette flow of a reactive third grade fluid with viscous dissipation, Int. Communication in heat and mass transfer 35, No.2 (2008) 188-196.

[23] M.S. Sharma, and , B. Nageswar Rao, Heat transfer in a visco-elastic fluid over a stretching sheet, J. of mathematical analysis and applications 222, No.1 (1998) 268-275. 
[24] B. Sahoo, S. Poncet, Flow and heat transfer of a third grade fluid past an exponentially stretching sheet with partial slip boundary condition, Int. J. of heat and mass transfer 54, No.23-24 (2011) 5010-5019.

[25] I. Teipel, Stagnation point flow of a Non-Newtonian second grade fluid, Transaction of the Canadian society for Mech. Engg. 12, No.2 (1988) 57-61.

[26] W.C. Tan, and T. Masuoka, Stokes first problem for a second grade fluid in a porous half space with heated boundary, Int. J. non-linear mech. 40, No.4 (2005) 515-522.

[27] K.Vajravelu and D. Rollins, Hydromagnetic flow of a second grade fluid over a stretching sheet, Applied mathematics and computations 148, No.3 (2004) 783-791. 